Classification

Physics Abstracts

$03.65-32.80-42.50-42.69$

\title{
Coherent population trapping and Fano profiles
}

\author{
B. Lounis and C. Cohen-Tannoudji
}

Laboratoire de Spectroscopie Hertzienne de l'E.N.S.( $\left(^{*}\right)$ and Collège de France, 24 rue Lhomond, F-75231 Paris Cedex 05, France

(Received 15 January 1992, accepted 23 January 1992)

\begin{abstract}
Résumé - - Cet article présente une nouvelle approche au phénomène de piégeage cohérent de population observable sur un système atomique à 3 niveaux en configuration $\Lambda$. Cette approche, basée sur la théorie de la diffusion, est valable lorsqu'un des deux champs lasers excitant le système atomique est beaucoup plus faible que l'autre. L'amplitude de diffusion du champ faible apparaît comme la somme de deux amplitudes résonnantes. Les positions et les largeurs des deux résonances correspondantes sont calculées et interprétées physiquement, à la limite des faibles saturations, en termes de diffusion Rayleigh et de diffusion Raman stimulée et spontanée. On montre enfin que l'interférence entre ces deux amplitudes de diffusion fait apparaître des profils de Fano dans les courbes donnant les variations en fréquence de la section efficace totale de diffusion.
\end{abstract}

\begin{abstract}
This paper presents a new approach to coherent population trapping in a $\Lambda$-type three level atomic configuration. This approach, which is based on scattering theory, applies when one of the two driving laser fields is much weaker than the other one. We show that the scattering amplitude of the weak field is the sum of 2 resonant amplitudes. The positions and the widths of these resonances are identified and physically interpreted in the low saturation limit, in terms of Rayleigh scattering, stimulated and spontaneous Raman scattering. Finally, we show that the interference between the two scattering amplitudes gives rise to Fano profiles in the curves giving the frequency dependence of the total scattering cross-section.
\end{abstract}

\section{Introduction.}

We consider in this paper an atomic system with three levels, $e, g_{1}, g_{2}$, forming a $\Lambda$-configuration (Fig. 1). The two transitions $g_{1}-e$ and $g_{2}-e$ are driven by two laser fields with frequencies $\omega_{L 1}$ and $\omega_{L 2}$ close, respectively, to the atomic frequencies $\omega_{e 1}=\left(E_{e}-E_{g 1}\right) / \hbar$ and $\omega_{e 2}=\left(E_{e}-E_{g 2}\right) / \hbar$. We denote :

(') Laboratoire associé au C.N.R.S. et à l'Université Pierre et Marie Curie. 


$$
\delta_{1}=\omega_{L 1}-\omega_{e 1} \quad \delta_{2}=\omega_{L 2}-\omega_{e 2}
$$

the corresponding detunings between the laser and atomic frequencies. The atom can decay from $e$ to $g_{1}$ and from $e$ to $g_{2}$ by spontaneous emission with rates respectively equal to $\Gamma_{1}$ and $\Gamma_{2}$. We put :

$$
\Gamma=\Gamma_{1}+\Gamma_{2}
$$

We assume that $g_{2}$ is above $g_{1}\left(E_{g_{2}}>E_{g_{1}}\right)$, but that no spontaneous emission can occur from $g_{2}$ to $g_{1}$.

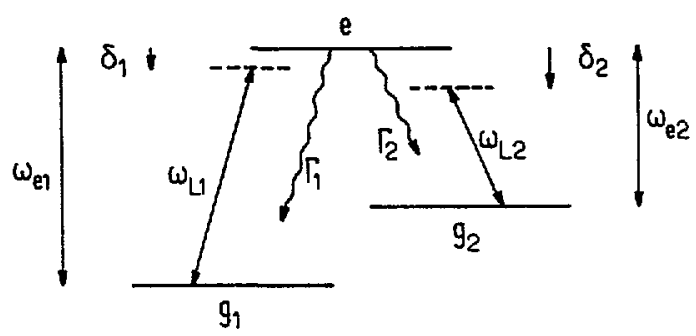

Fig. 1. - Three level $\Lambda$-configuration $\left\{e, g_{1}, g_{2}\right\}$ driven by two laser fields at frequencies $\omega_{L 1}$ and $\omega_{L 2} . \delta_{1}=\omega_{L 1}-\omega_{e 1}$ and $\delta_{2}=\omega_{L 2}-\omega_{e 2}$ are the two detunings; $\Gamma_{1}$ and $\Gamma_{2}$ are, respectively, the two spontaneous emission rates from $e$ to $g_{1}$ and $g_{2}$.

Such a configuration gives rise to the phenomenon of "coherent population trapping" [1, 2]. When the two detunings $\delta_{1}$ and $\delta_{2}$ are equal, i.e. when the resonance Raman condition :

$$
\hbar \omega_{L 1}-\hbar \omega_{L 2}=E_{g_{2}}-E_{g_{1}}
$$

between the two states $g_{1}$ and $g_{2}$ is fulfilled, the steady-state population $\sigma_{e e}^{\text {st }}$ of the upper state vanishes and the fluorescence stops. Several theoretical treatments have been given for such an effect [3-6]. The main result is that atoms are optically pumped in a linear superposition of the two lower states which is not coupled to the laser light because the two absorption amplitudes from $g_{1}$ to $e$ and from $g_{2}$ to $e$ interfere destructively. Several applications of coherent population trapping have been developed, including high resolution spectroscopy [7], subrecoil laser cooling [8], adiabatic transfer of populations [9], amplification without inversion [10].

Suppose that $\omega_{L 2}$ is fixed and that $\omega_{L 1}$ is scanned. The variations with $\omega_{L 1}$, or equivalently with $\delta_{1}=\omega_{L 1}-\omega_{e 1}$, of $\sigma_{e e}^{\text {st }}$ may be studied from the steady-state solution of optical Bloch equations, which can be determined analytically and which may be found for example in reference [5].

$$
\sigma_{e e}^{\mathrm{st}}=\frac{4\left(\delta_{1}-\delta_{2}\right)^{2} \Omega_{1}^{2} \Omega_{2}^{2} \Gamma}{Z}
$$

where

$$
\begin{aligned}
Z & =8\left(\delta_{1}-\delta_{2}\right)^{2} \Omega_{1}^{2} \Omega_{2}^{2} \Gamma+4\left(\delta_{1}-\delta_{2}\right)^{2} \Gamma^{2}\left(\Omega_{1}^{2} \Gamma_{2}+\Omega_{2}^{2} \Gamma_{1}\right) \\
& +16\left(\delta_{1}-\delta_{2}\right)^{2}\left[\delta_{1}^{2} \Omega_{2}^{2} \Gamma_{1}+\delta_{2}^{2} \Omega_{1}^{2} \Gamma_{2}\right]-8 \delta_{1}\left(\delta_{1}-\delta_{2}\right) \Omega_{2}^{4} \Gamma_{1} \\
& +8 \delta_{2}\left(\delta_{1}-\delta_{2}\right) \Omega_{1}^{4} \Gamma_{2}+\left(\Omega_{1}^{2}+\Omega_{2}^{2}\right)^{2}\left(\Omega_{1}^{2} \Gamma_{2}+\Omega_{2}^{2} \Gamma_{1}\right)
\end{aligned}
$$

In (1.4) and (1.5), $\Omega_{1}$ and $\Omega_{2}$ are the $R$ abi frequencies characterizing, respectively, the couplings of the two laser fields at $\omega_{L 1}$ and $\omega_{L 2}$ with the transitions $g_{1}-e$ and $g_{2}-e$. The variations with 
$\delta_{1}$ of $\sigma_{e e}^{\text {st }}$ are represented in figure 2 , in the limiting case where $\Omega_{2} \gg \Omega_{1}$. We have supposed $\delta_{2}=-1.5 \Gamma$. They clearly exhibit two resonances : a broad one, near $\delta_{1}=0$, and a narrow one near $\delta_{1}=\delta_{2}$, where $\sigma_{e e}^{\text {st }}$ vanishes. The narrow structure of figure 2 is quite similar to the Fano profiles which can be observed when a discrete state is coupled to a continuum via two channels, directly and through a discrete state embedded in this continuum [11]. Well known examples of such profiles are found in autoionizing resonances [11] and in laser induced continuum structures [12 - 13].

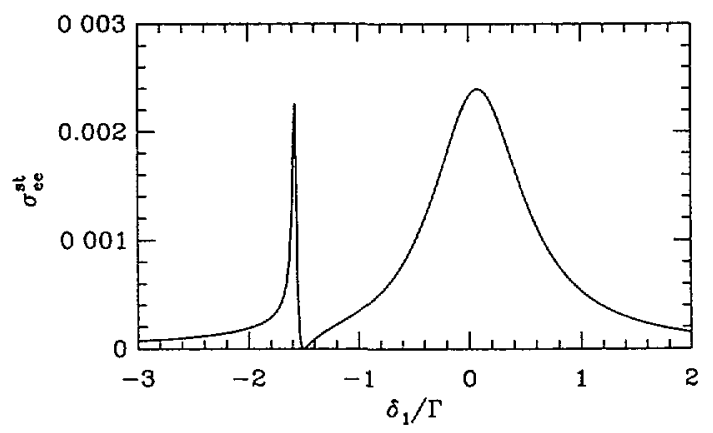

Fig. 2. - Steady-state population of the excited state, calculated from optical Bloch equations, versus $\delta_{1}$ in units of $\Gamma . \delta_{2}$ is fixed and equal to $-1.5 \Gamma, \Omega_{2}=0.7 \Gamma, \Omega_{1}=0.025 \Gamma$. Two resonances are clearly visible, the narrow one looking like a Fano profile.

The purpose of this paper is to investigate the connections which exist between coherent population trapping in a $\Lambda$-configuration and Fano profiles. Such a connection cannot be easily analyzed from expressions such as (1.4) and (1.5) which have not a transparent physical meaning. We prefer to adopt here another point of view which, we hope, can provide new physical insights in coherent population trapping. We consider the scattering of a single photon $\omega_{L 1}$ by the atom interacting with several $\omega_{L 2}$ photons and we try to identify in the total scattering amplitude the various physical paths followed by the system and whose interference can give rise to structures such as the one appearing in figure 2. The paper is organized as follows. We first specify in section 2 our assumptions on the scattering process considered in this paper. A non-perturbative expression for the scattering amplitude is then derived in section 3 and its general properties are analyzed. The limiting case where the transition $g_{2}-e$ is not saturated by the $\omega_{L 2}$ photons is investigated in section 4 and this allows us to identify the physical processes associated with the two resonances of figure 2 . Finally, we show in section 5 how Fano profiles can be associated with the narrow structure of figure 2.

\section{Scattering process considered in this paper.}

2.1 INITIAL STATE AND FINAL STATE. - In the initial state :

$$
|i\rangle=\left|g_{1} ;(1)_{1},(N)_{2},(0)_{j}\right\rangle
$$

of the scattering process, the atom is in $g_{1}$, in the presence of 1 photon $\omega_{L 1}, N$ photons $\omega_{L 2}$, all other field modes $j$ (with $j \neq 1,2$ ) being empty. We consider here the scattering process 
leading to the final state :

$$
|f\rangle=\left|g_{1} ;(0)_{1},(N)_{2},(1)_{\omega}\right\rangle
$$

where the atom is still in $g_{1}$, whereas the incident photon $\omega_{L 1}$ has disappeared and has been replaced by one fluorescence photon $\omega$.

The final state (2.2) is not the only possible one. Other scattering processes, involving several fluorescence photons and the absorption of one or several $\omega_{L_{2}}$ photons could also be considered. For each of these possible scattering processes, starting from (2.1), the atom has first to go from $g_{1}$ to $e$, and one can show that the total cross-section for each of these scattering processes is proportional to the steady-state population of e. Since we are mainly interested here in $\sigma_{e e}^{\mathrm{st}}$, we restrict ourselves to the simplest possible scattering process, the one leading from (2.1) to (2.2). We will check in subsection 3.4 that the total scattering cross section for such a process is proportional to $\sigma_{e e}^{\text {st }}$. Note also that $g_{2}$ cannot appear in the final state of a scattering process. Photons $\omega_{L 2}$ can indeed be absorbed by the atom in $g_{2}$, so that $g_{2}$ is an unstable state.

When the radiation field is quantized in a box of volume $L^{3}$, the Rabi frequencies $\Omega_{1}$ and $\Omega_{2}$ associated with (2.1) are respectively proportional to :

$$
\Omega_{1} \sim \sqrt{\frac{\hbar \omega_{L 1}}{2 \varepsilon_{0} L^{3}}} \quad \Omega_{2} \sim \sqrt{\frac{N \hbar \omega_{L 2}}{2 \varepsilon_{0} L^{3}}}
$$

The fact that $\Omega_{1}$ tends to zero when $L \longrightarrow \infty$ does not raise any difficulty. The flux associated with the incident photon $\omega_{L 1}$ is equal to $c / L^{3}$ and the ratio of the transition rate, proportional to $\Omega_{1}^{2}$, i.e. to $1 / L^{3}$, by the incident flux $c / L^{3}$, is independent of $L$, as expected for a physical quantity such as a scattering cross section. The situation is different for $\Omega_{2}$. When $L$ tends to infinity, we must also let $N$ tend to infinity, keeping $N / L^{3}$ constant, in order to have $\Omega_{2}$ fixed.

2.2 ASSUMPTIONS CONCERNING THE INTERACTION HAMILTONIAN V, - The Hamiltonian $\mathrm{H}$ of the total system "atom + photons" is equal to :

$$
\mathrm{H}=\mathrm{H}_{\mathbf{0}}+\mathrm{V}
$$

where $\mathrm{V}$ is the photon-atom interaction Hamiltonian and where $H_{0}$ is the sum of the energies of the non interacting systems.

In the electric-dipole and rotating-wave approximations, $\mathrm{V}$ can be written :

$$
\mathbf{V}=-\mathbf{d}^{+} \cdot \mathbf{E}^{+}-\mathbf{d}^{-} \mathbf{E}^{-}
$$

where $\mathrm{d}^{+}$and $\mathrm{d}^{-}$are, respectively, the raising and lowering parts of the dipole operator, and where $\mathbf{E}^{+}$and $\mathbf{E}^{-}$are, respectively, the positive and negative frequency components of the electric field operator. With such an interaction Hamiltonian, the only processes which can take place when the atom is in $g_{1}$, is the absorption of one photon, the atom going from $g_{1}$ to $e$. We make here the further assumption that $\omega_{e 1}$ and $\omega_{e 2}$ are so different that it is legitimate to neglect the non resonant coupling of photons $\omega_{L 2}$ with the atom in $g_{1}$.

From the previous assumptions, it follows that $\mathrm{V}$ acting upon $|i\rangle$ can lead only to. $\mid e ;(0)_{1}$, $\left.(N)_{2},(0)_{j}\right)$. More precisely,

$$
V|i\rangle=\frac{\hbar \Omega_{1}}{2}\left|e ;(0)_{1},(N)_{2},(0)_{j}\right\rangle
$$

which is the precise definition of the Rabi frequency $\Omega_{1}$. Similarly,

$$
\mathrm{V}|f\rangle=\frac{\hbar \Omega}{2}\left|e ;(0)_{1},(N)_{2},(0)_{j}\right\rangle
$$

where $\Omega$ is the Rabi frequency characterizing the coupling with the fluorescence photon $\omega$. 


\section{Calculation of the scattering amplitude.}

3.1 General EXPRESSION OF THE $S$-MATRIX ELEMENT. - Since we neglect virtual emissions of photons by the atom in $g_{1}$ (rotating-wave approximation), the states $|i\rangle$ and $|f\rangle$ introduced above can be considered as correct asymptotic scattering states (see for example, Ref. [14], Complement $B_{I I I}$ ). The element $S_{\mathrm{f}}$ of the $S$-matrix, between the initial and final states, can then be written :

$$
S_{\mathrm{fi}}=\delta_{\mathrm{f}}-2 \pi i \delta\left(E_{\mathrm{f}}-E_{\mathrm{i}}\right) T_{\mathrm{fi}}
$$

where $E_{\mathrm{i}}$ and $E_{\mathrm{f}}$ are the unperturbed energies of $|i\rangle$ and $|f\rangle$ (which are eigenstates of $\mathrm{H}_{0}$ ), and where the transition matrix element $T_{\mathrm{fi}}$ is given by :

$$
T_{\mathrm{fi}}=\langle f|\mathrm{~V}| i\rangle+\lim _{\eta \rightarrow 0_{+}}\left\langle f\left|\mathrm{~V} \frac{1}{E_{\mathrm{i}}-H+i \eta} \mathrm{V}\right| i\right\rangle
$$

Note that it is $\mathrm{H}$, and not $\mathrm{H}_{0}$, which appears in the exact expression (3.2). Expanding the propagator $\left(E_{i}-\mathbf{H}+i \eta\right)^{-1}$ in powers of the unperturbed propagator $\left(E_{i}-\mathrm{H}_{0}+i \eta\right)^{-1}$ and $\mathrm{V}$ would give the Born expansion of the scattering matrix. We don't make such an expansion here. We keep the exact expression (3.2), in order to get non perturbative scattering amplitudes including the shift and the broadening of the intermediate states appearing in the scattering process.

3.2 CONNECTION BETWEEN THE SCATTERING-MATRIX AND THE RESOLVENT $G(z)$ OF THE HAMILTONIAN. - In order to connect $|i\rangle$ to $|f\rangle$, one must destroy the incident photon $\omega_{L 1}$ and create the scattered photon $\omega$. The interaction Hamiltonian $\mathrm{V}$, given in (2.5), can only destroy or create a single photon at a time. It follows that :

$$
\langle f|\mathrm{~V}| \boldsymbol{i}\rangle=0
$$

Using (2.6) and (2.7), we can then transform (3.2) into :

$$
T_{\mathrm{fi}}=\frac{\hbar^{2} \Omega \Omega_{1}}{4} \lim _{\eta \rightarrow 0_{+}}\left\langle\varphi_{e}\left|G\left(z=E_{\mathrm{i}}+i \eta\right)\right| \varphi_{e}\right\rangle
$$

where :

$$
\mathrm{G}(z)=\frac{1}{z-\mathbf{H}}
$$

is the resolvent of the Hamiltonian $\mathrm{H}, z$ being a complex variable, and where we have introduced the simplified notation :

$$
\left|\varphi_{e}\right\rangle=\left|e ;(0)_{1},(N)_{2},(0)_{j}\right\rangle
$$

3.3 Calculation of the relevant matrix Elements of the Resolvent. - The atom, in $e$, can spontaneously emit a photon and decay to $g_{1}$ or $g_{2}$. The state $\left|\varphi_{e}\right\rangle$, given in (3.6), is thus coupled to continua and radiatively unstable. Well known projection operator techniques are available for calculating the diagonal element $G_{e e}(z)=\left\langle\varphi_{e}|G(z)| \varphi_{e}\right\rangle$ (see for example Ref. [14], Chap. III). Before doing such a calculation, one must not forget however to check if there are no other discrete states of $H_{0}$, close to $\left|\varphi_{e}\right\rangle$, and which would be coupled to $\left|\varphi_{e}\right\rangle$ or to the same continua as $\left|\varphi_{e}\right\rangle$. In such a case, it is well known that the decay of $\left|\varphi_{e}\right\rangle$ cannot 
be studied independently of these other discrete states. Simple expressions for the matrix elements of $\mathrm{G}(z)$ can be obtained only if one considers the projection of $\mathrm{G}(z)$ onto the subspace $\mathcal{E}_{0}$ subtended by the discrete states of $\mathrm{H}_{0}$ which are coupled directly or indirectly through the same continua.

Actually, in the problem considered in this paper, we have another discrete state of $\mathrm{H}_{0}$

$$
\left|\varphi_{2}\right\rangle=\left|g_{2} ;(0)_{1},(N+1)_{2},(0)_{j}\right\rangle
$$

which is coupled to $\left|\varphi_{e}\right\rangle$ since the atom in $g_{2}$ can absorb one $\omega_{L 2}$ photon and jump to $e$. By definition of the Rabi frequency $\Omega_{2}$, we have indeed :

$$
\left\langle\varphi_{e}|\mathrm{~V}| \varphi_{2}\right\rangle=\left\langle e ;(0)_{1},(N)_{2},(0)_{j}|\mathrm{~V}| g_{2} ;(0)_{1},(N+1)_{2},(0)_{j}\right\rangle=\frac{\hbar \Omega_{2}}{2}
$$

Furthermore, the unperturbed energies $E_{\varphi_{e}}$ and $E_{\varphi_{2}}$ of $\left|\varphi_{e}\right\rangle$ and $\left|\varphi_{2}\right\rangle$ are close to each other. If we measure the unperturbed energies relative to $E_{\varphi_{e}}$, by taking :

$$
E_{\varphi_{e}}=0
$$

then, we have :

$$
E_{\varphi_{2}}=\hbar \delta_{2}
$$

Strictly speaking, one should also consider the initial state $|i\rangle$ given in (2.1), which is coupled to $\left|\varphi_{e}\right\rangle$, since, according to (2.6),

$$
\left\langle\varphi_{e}|V| i\right\rangle=\frac{\hbar \Omega_{1}}{2}
$$

This state has an energy $E_{\mathrm{i}}$ close to $E_{\varphi_{e}}=0$

$$
E_{\mathrm{i}}=\hbar \delta_{1}
$$

One must not forget however that, in the limit $L \longrightarrow \infty, \Omega_{1}$ tends to zero (whereas $\Omega_{2}$ keeps the same value), so that we can neglect the influence of $|i\rangle$ on the decay of $\left|\varphi_{e}\right\rangle$.

The previous discussion shows that we must introduce a two dimensional subspace $\mathcal{E}_{0}$ of eigenstates of $\mathrm{H}_{0}$

$$
\mathcal{E}_{0}=\left\{\left|\varphi_{e}\right\rangle,\left|\varphi_{2}\right\rangle\right\}
$$

and study $\mathrm{PG}(z) \mathrm{P}$, where $\mathrm{P}$ is the projector onto this subspace. One can then show (see, for example, Ref. [14], Chap. III) that, when $z$ is close to $E_{\mathrm{i}}+i \eta, \mathrm{PG}(z) \mathrm{P}$ can be considered as the resolvent of an effective Hamiltonian $\mathrm{H}_{\mathrm{eff}}$.

$$
\mathrm{PG}(z) \mathrm{P}=\frac{\mathrm{P}}{z-\mathrm{H}_{\mathrm{eff}}}
$$

Such a Hamiltonian governs the evolution of the system within $\mathcal{E}_{0}$ and is represented by the following $2 \times 2$ matrix :

$$
\left(\mathrm{H}_{\mathrm{eff}}\right)=\hbar\left(\begin{array}{cc}
-i \Gamma / 2 & \Omega_{2} / 2 \\
\Omega_{2} / 2 & \delta_{2}
\end{array}\right)
$$

Note that $\mathrm{H}_{\text {eff }}$ differs from $\mathrm{PH}_{0} \mathrm{P}+\mathrm{PVP}$ only by an imaginary term, $-i \hbar \Gamma / 2$, added to the energy of the excited state $e$, and describing the radiative unstability of this state. 
Written in matrix form, the operator equation (3.14) becomes for $z=E_{\mathrm{i}}+i \eta=\hbar \delta_{1}+i \eta$, according to (3.12) :

$$
\left(\begin{array}{ll}
G_{e e}\left(\hbar \delta_{1}+i \eta\right) & G_{2 e}\left(\hbar \delta_{1}+i \eta\right) \\
G_{e 2}\left(\hbar \delta_{1}+i \eta\right) & G_{e e}\left(\hbar \delta_{1}+i \eta\right)
\end{array}\right)=\frac{1}{\hbar}\left(\begin{array}{cc}
\delta_{1}+i \frac{\Gamma}{2} & -\frac{\Omega_{2}}{2} \\
-\frac{\Omega_{2}}{2} & \delta_{1}-\delta_{2}
\end{array}\right)^{-1}
$$

Since the two eigenvalues of $\mathrm{H}_{\text {eff }}$ are complex, we have not kept i $\eta$ in the matrix appearing in right-hand side of (3.16). Taking the inverse of this matrix, and using (3.4) and (3.12), we get :

$$
T_{\mathrm{f}}=\frac{\hbar \Omega_{1} \Omega}{4} \frac{\delta_{1}-\delta_{2}}{\mathcal{D}}
$$

where

$$
\mathcal{D}=\left(\delta_{1}+i \frac{\Gamma}{2}\right)\left(\delta_{1}-\delta_{2}\right)-\frac{\Omega_{2}^{2}}{4}=\delta_{1}\left(\delta_{1}-\delta_{2}\right)-\frac{\Omega_{2}^{2}}{4}+i \frac{\Gamma}{2}\left(\delta_{1}-\delta_{2}\right)
$$

is the determinant of the matrix $\delta_{1}-H_{\text {eff }} / \hbar$. Introducing the eigenvalues $\hbar z_{\mathbf{I}}$ and $\hbar z_{\mathrm{II}}$ of $H_{\text {eff }}$, we can also write :

$$
\mathcal{D}=\left(\delta_{1}-z_{\mathrm{I}}\right)\left(\delta_{1}-z_{\mathrm{II}}\right)
$$

which, inserted into (3.17), gives :

$$
T_{\mathrm{f}}=\frac{\hbar \Omega_{1} \Omega}{4} \frac{\delta_{1}-\delta_{2}}{\left(\delta_{1}-z_{\mathrm{I}}\right)\left(\delta_{1}-z_{\mathrm{II}}\right)}=\frac{\hbar \Omega_{1} \Omega}{4\left(z_{\mathrm{I}}-z_{\mathrm{II}}\right)}\left[\frac{z_{\mathrm{I}}-\delta_{2}}{\delta_{1}-z_{\mathrm{I}}}-\frac{z_{\mathrm{II}}-\delta_{2}}{\delta_{1}-z_{\mathrm{II}}}\right]
$$

3.4 General PRoperties of The SCATtering amplitude. - Considered as a function of $\delta_{1}$, the scattering amplitude $T_{\mathrm{f}}$ appears in (3.20) as a sum of two resonant scattering amplitudes : one centered about $\delta_{1}=\operatorname{Re} z_{\mathrm{I}}$, with a width equal to Im $z_{\mathrm{I}}$; and the other one centered about $\delta_{1}=\operatorname{Re} z_{\text {II }}$, with a width equal to $\operatorname{Im} z_{I I}$. The theoretical approach followed in this paper clearly shows that two resonances should appear in the variation with $\delta_{1}$ of the total scattering cross-section (which is proportional to $\left|T_{\mathrm{f}}\right|^{2}$ ), and it relates the positions and the widths of these resonances to the real and imaginary parts of the eigenvalues of $H_{\text {eff }}$.

It also clearly appears from (3.17) that $T_{\mathrm{f}}$ vanishes for $\delta_{1}=\delta_{2}$. The quenching of the fluorescence, when the resonance Raman condition between $g_{1}$ and $g_{2}$ is fulfilled, is also predicted by such an approach.

Let us finally calculate $\left|T_{\mathrm{f}}\right|^{2}$ Using (3.17) and (3.18), we get :

$$
\left|T_{\mathrm{f}}\right|^{2}=\frac{\hbar^{2} \Omega_{1}^{2} \Omega^{2}}{16} \frac{\left(\delta_{1}-\delta_{2}\right)^{2}}{\left[\delta_{1}\left(\delta_{1}-\delta_{2}\right)-\frac{\Omega_{2}^{2}}{4}\right]^{2}+\frac{\Gamma^{2}}{4}\left(\delta_{1}-\delta_{2}\right)^{2}}
$$

It is interesting now to compare (3.21) with the limit of (1.4) when $\Omega_{2} \gg \Omega_{1}$. Since $\Omega_{1}^{2}$ already appears in the numerator of $(1.4)$, we can put $\Omega_{1}=0$ in $Z$. One can then easily check that $\left|T_{\mathrm{f}}\right|^{2}$ and $\sigma_{e e}^{\mathrm{st}}$ are proportional, and have therefore the same dependence in $\delta_{1}, \delta_{2}, \Omega_{2}$. As expected, we find that the total scattering cross-section, for the process $|i\rangle \rightarrow|f\rangle$ considered in this paper, involves the steady-state population of $e$. In the limit $\Omega_{1} \ll \Omega_{2}$, we can therefore get new physical insights in equations (1.4) and (1.5), and in the variations with $\delta_{1}$ of $\sigma_{e e}^{\text {st }}$ represented in figure 2 , by considering the two resonant scattering amplitudes appearing in (3.20). 


\section{The low saturation limit. Physical discussion.}

The calculations of the previous section 3 are valid for any value of $\Omega_{2}$ and $\delta_{2}$. We consider now the limit where $\Omega_{2}$ is small compared to $\Gamma$ or to $\left|\delta_{2}\right|$. More precisely, we suppose that the saturation parameter $s_{2}$ for the transition $g_{2}-e$ is small compared to 1 :

$$
s_{2}=\frac{\Omega_{2}^{2} / 2}{\delta_{2}^{2}+\frac{\Gamma^{2}}{4}} \ll 1
$$

Such a limit is interesting because it leads to two eigenvalues of $\mathrm{H}_{\mathrm{eff}}, z_{\mathrm{I}}$ and $z_{\mathrm{II}}$, having quite different imaginary parts. The corresponding resonances appearing in the variations of $T_{\mathrm{f}}$ with $\delta_{1}$ have then quite different widths, which is indeed the situation leading to Fano profiles.

4.1 INTERPRETATION OF THE TWO RESONANCES APPEARING IN THE SCATTERING AMPLITUDE. - Let $z_{11}$ be the eigenvalue of $\mathrm{H}_{\text {eff }} / \hbar$ which tends to $\delta_{2}$ when $\Omega_{2}$ tends to zero. One can always write :

$$
z_{\mathrm{II}}=\delta_{2}+\delta_{2}^{\prime}-i \frac{\Gamma_{2}^{\prime}}{2}
$$

where $\delta_{2}^{\prime}$ and $-i \Gamma_{2}^{\prime} / 2$ are the real and imaginary parts of the correction introduced by $\Omega_{2}$. Because the trace of $\mathrm{H}_{\text {eff }}$ is invariant in a change of basis, we have for the other eigenvalue $z_{\mathrm{I}}$, which tends to $-i \Gamma / 2$ when $\Omega_{1}$ tends to zero:

$$
z_{\mathrm{I}}=-\delta_{2}^{\prime}-i \frac{\Gamma-\Gamma_{2}^{\prime}}{2}
$$

Condition (4.1) allows one to calculate $\delta_{2}^{\prime}$ and $\Gamma_{2}^{\prime}$ perturbatively. One gets :

$$
\delta_{2}^{\prime}-i \frac{\Gamma_{2}^{\prime}}{2}=\frac{\left(\Omega_{2} / 2\right)^{2}}{\delta_{2}+i \frac{\Gamma}{2}}
$$

which gives :

$$
\begin{aligned}
\delta_{2}^{\prime} & =\delta_{2} \frac{s_{2}}{2} \\
\Gamma_{2}^{\prime} & =\Gamma \frac{s_{2}}{2}
\end{aligned}
$$

Equation (4.5a) shows that $\delta_{2}^{\prime}$ is the light shift of level $g_{2}$ due to the coupling with the photons $\omega_{L 2}$, whereas equation (4.5b) shows that $\Gamma_{2}^{\prime}$ is the radiative broadening of level $g_{2}$, or equivalently the departure rate from level $g_{2}$, due to the absorption of $\omega_{L 2}$ photons. Note that, although $\delta_{2}^{\prime}$ and $\Gamma_{2}^{\prime}$ are calculated here perturbatively, their presence in the denominator of the two fractions of (3.20) corresponds to a nonperturbative expression for $T_{\mathrm{f}}$. Finally, equation (4.3) shows that the light shift of level $e$ is opposite to that of level $g_{2}$, whereas the radiative width of level $e$ is slightly reduced from $\Gamma$ to $\Gamma-\Gamma_{2}^{\prime}$ as a result of the contamination of $e$ by $g_{2}$ induced by $\Omega_{2}$.

The physical meaning of $\delta_{2}^{\prime}$ and $\Gamma_{2}^{\prime}$ can now be used to interpret the two resonances appearing in (3.22). Consider first the resonance associated with the denominator $\delta_{1}-z_{\mathrm{I}}$. The position of its center is given by :

$$
\delta_{1}=\operatorname{Re} z_{\mathrm{I}}=-\delta_{2}^{\prime}
$$


an equation which can be also written :

$$
\hbar \omega_{L 1}=E_{e}-\hbar \delta_{2}^{\prime}-E_{g_{1}}
$$

and which expresses an optical resonance condition between the lower level $g_{1}$ and the lightshifted upper level $e$. As expected, the width of this optical resonance, $\operatorname{Im} z_{I}=\left(\Gamma-\Gamma^{\prime}\right) / 2 \simeq \Gamma / 2$, is mainly determined by the natural width $\Gamma$ of $e$. Consider now the second resonance associated with the denominator $\delta_{1}-z_{11}$. Its position is determined by :

$$
\delta_{1}=\operatorname{Re} z_{1 I}=\delta_{2}+\delta_{2}^{\prime}
$$

and corresponds to :

$$
\hbar \omega_{L 1}-\hbar \omega_{L 2}=E_{g_{2}}+\hbar \delta_{2}^{\prime}-E_{g_{1}}
$$

Equation (4.9) expresses a stimulated Raman resonance condition between the light-shifted level $g_{2}$ and the level $g_{1}$. Note the difference between (4.9) and (1.3), which is the stimulated Raman resonance condition between unperturbed energy levels (without light shifts) leading to coherent population trapping. As for the width of the resonance associated with $z_{11}$, it is determined by the radiative width $\Gamma_{2}^{\prime}$ of level $g_{2}$ due to the absorption of $\omega_{L 2}$ photons. For small $s_{2}$, this width is much smaller than $\Gamma$ (see (4.5b)), so that the resonance associated with $z_{I I}$ is much narrower than the resonance associated with $z_{I}$.

4.2 The INTERMEdite STATES OF THE SCATTERING PROCESS. - From the results of subsection 3.2 , it follows that the scattering amplitude $T_{\mathrm{f}}$ can be written :

$$
T_{\mathrm{f}}=\frac{\hbar^{2} \Omega_{1} \Omega}{4}\left\langle\varphi_{e}\left|\frac{1}{\hbar \delta_{1}-H_{\text {eff }}}\right| \varphi_{e}\right\rangle
$$

Let $\overline{\left|\varphi_{e}\right\rangle}$ and $\overline{\left|\varphi_{2}\right\rangle}$ be the eigenvectors of $H_{\text {eff }}$ which tend to $\left|\varphi_{e}\right\rangle$ and $\left|\varphi_{2}\right\rangle$ when $\Omega_{2}$ tends to zero. A perturbative expansion can be given for $\overline{\left|\varphi_{e}\right\rangle}$ and $\overline{\left|\varphi_{2}\right\rangle}$. Using (3.15), we get :

$$
\begin{aligned}
& \overline{\left|\varphi_{e}\right\rangle} \simeq\left|\varphi_{e}\right\rangle-\frac{\Omega_{2} / 2}{\delta_{2}+i(\Gamma / 2)}\left|\varphi_{2}\right\rangle \\
& \overline{\left|\varphi_{2}\right\rangle} \simeq\left|\varphi_{2}\right\rangle+\frac{\Omega_{2} / 2}{\delta_{2}+i(\Gamma / 2)}\left|\varphi_{e}\right\rangle
\end{aligned}
$$

Note that $\overline{\left|\varphi_{e}\right\rangle}$ and $\overline{\left|\varphi_{2}\right\rangle}$ are not orthogonal, since $H_{\text {eff }}$ is not hermitian. They form however a basis in the subspace $\mathcal{E}_{0}$, and we can always expand $\left|\varphi_{e}\right\rangle$ on such a basis :

$$
\left|\varphi_{e}\right\rangle=\alpha \overline{\left|\varphi_{e}\right\rangle}+\beta \overline{\left|\varphi_{2}\right\rangle}
$$

the non orthogonality of $\overline{\left|\varphi_{e}\right\rangle}$ and $\overline{\left|\varphi_{2}\right\rangle}$ resulting in the fact that the components $\alpha$ and $\beta$ of $\left|\varphi_{e}\right\rangle$ do not coincide respectively with the scalar products $\left.\overline{\left\langle\varphi_{e}\right|} \varphi_{e}\right\rangle$ and $\left.\overline{\left\langle\varphi_{2}\right|} \varphi_{e}\right\rangle$. Inserting (4.12) into (4.10) and using the fact that $\overline{\left|\varphi_{e}\right\rangle}$ and $\overline{\left|\varphi_{2}\right\rangle}$ are eigenstates of $H_{\text {eff }}$ with eigenvalues $\hbar z_{\mathrm{I}}$ and $\hbar z_{\mathrm{II}}$, we get :

$$
T_{\mathrm{f}}=\frac{\hbar \Omega_{1} \Omega}{4}\left\{\alpha \left\langle\varphi_{e} \overline{\left|\varphi_{e}\right\rangle} \frac{1}{\delta_{1}-z_{1}}+\beta\left\langle\varphi_{e} \overline{\left|\varphi_{2}\right\rangle} \frac{1}{\delta_{1}-z_{I I}}\right\}\right.\right.
$$




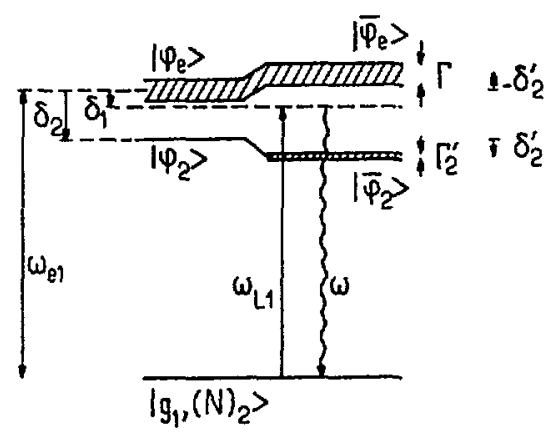

Fig. 3. - Diagrammatic representation of the scattering process in terms of dressed states. Starting from $\left|g_{1},(N)_{2}\right\rangle$, the dressed atom absorbs the incident $\omega_{L 1}$ photon (upward arrow), jumps to one of the two dressed states $\overline{\left|\varphi_{e}\right\rangle}$ or $\overline{\left|\varphi_{2}\right\rangle}$ originating from $\left|\varphi_{e}\right\rangle=\left|e,(N)_{2}\right\rangle$ and $\left|\varphi_{2}\right\rangle=\left|g_{2},(N+1)_{2}\right\rangle$, and then falls back to $\left|g_{1},(N)_{2}\right\rangle$ by emitting the fluorescence photon $\omega$ (downward wavy arrow).

Such a result shows that, after the absorption of the incident photon $\omega_{L 1}$, and before the emission of the fluorescence photon $\omega$, the system passes through two possible intermediate states, which are the eigenstates $\overline{\left|\varphi_{e}\right\rangle}$ and $\overline{\left|\varphi_{2}\right\rangle}$ of $H_{\text {eff }}$.

These two states can be also considered as two dressed states of the "atom $+\omega_{L 2}$ photons" system, as shown in figure 3. The left part of this figure represents a few unperturbed states of such a system : the two states $\left|\varphi_{e}\right\rangle=\left|e,(N)_{2}\right\rangle$ and $\left|\varphi_{2}\right\rangle=\left|g_{2},(N+1)_{2}\right\rangle$, which are separated by an interval $\delta_{2}$ (in angular frequency units), the state $\left|\varphi_{e}\right\rangle$ having a natural width $\Gamma$; the state $\left|g_{1},(N)_{2}\right\rangle$, which is located at a distance $\omega_{e 1}$ below $\left|\varphi_{2}\right\rangle$. When the coupling $\Omega_{2} / 2$ between $\left|\varphi_{2}\right\rangle$ and $\left|\varphi_{e}\right\rangle$ is taken into account, these two states repel each other by an amount $\delta_{2}^{\prime}$, which is the

light shift of $g_{2}$, and transform into two dressed states $\overline{\left|\varphi_{e}\right\rangle}$ and $\overline{\left|\varphi_{2}\right\rangle}$ which are represented in the right part of figure 3 . The contamination of the wave functions induced by $\Omega_{2}$ is responsible for the appearance of a small width $\Gamma_{2}^{\prime}$ for $\overline{\left|\varphi_{2}\right\rangle}$, whereas the width of $\overline{\left|\varphi_{e}\right\rangle}$ is slightly reduced. As for the state $\left|g_{1},(N)_{2}\right\rangle$, it remains unperturbed, since we neglect the non resonant coupling of the atom in $g_{1}$ with the $\omega_{L 2}$ photons. The scattering of the incident photon $\omega_{L 1}$ by such a system can then be considered as an elastic scattering process, where the dressed atom, starting from $\left|g_{1},(N)_{2}\right\rangle$, absorbs the incident photon $\omega_{L 1}$ (upward arrow of Fig. 3), jumps to $\overline{\left|\varphi_{2}\right\rangle}$ or $\overline{\left|\varphi_{e}\right\rangle}$, and then falls back to $\left|g_{1},(N)_{2}\right\rangle$ by emitting the fluorescence photon $\omega$ (downward wavy arrow). Another possible diagrammatic representation of such a process is given in figure 4, which represents more clearly than figure 3 the two possible intermediate states between the initial and final states. Note however than the energy defects in the two intermediate states are more visible on the diagrammatic representation of figure 3 , where they are just equal to the interval between the dotted line (reached after the absorption of the $\omega_{L 1}$ photon) and the energy of each dressed state $\overline{\left|\varphi_{e}\right\rangle}$ or $\overline{\left|\varphi_{2}\right\rangle}$, the width of each dressed state being included as an imaginary part of its energy.

The approach followed in this subsection could be easily extended to describe multiphoton scattering processes where several fluorescence photons are emitted and one or several $\omega_{L 2}$ photons are absorbed. For example, the dressed atom in $\left.\mid g_{1},(N)_{2}\right)$ can absorb the incident 


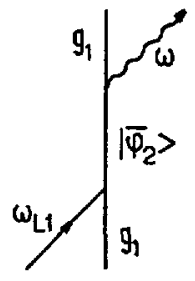

a)

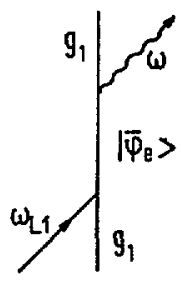

b)

Fig. 4. - Another diagrammatic representation of the scattering process showing the two possible intermediate states $\overline{\left|\varphi_{2}\right\rangle}$ and $\overline{\left|\varphi_{e}\right\rangle}$.

photon $\omega_{L 1}$, jump to one of the two dressed states $\overline{\left\langle\varphi_{e}^{N}\right\rangle}$ or $\overline{\left|\varphi_{2}^{N}\right\rangle}$ originating from the manifold $\left\{\left|e,(N)_{2}\right\rangle, \quad\left|g_{2},(N+1)_{2}\right\rangle\right\}$, then emit a fluorescence photon $\omega$, and fall into one of the two dressed states $\overline{\left|\varphi_{e}^{N-1}\right\rangle}$ and $\overline{\left|\varphi_{2}^{N-1}\right\rangle}$ originating from the manifold $\left\{\left|e,(N-1)_{2}\right\rangle,\left|g_{2},(N)_{2}\right\rangle\right\}$, and finally end into $\left|g_{1},(N-1)_{2}\right\rangle$ by emission of a second fluorescence photon $\omega "$. There are then four scattering amplitudes corresponding to four possible paths and one can show that, for $\delta_{1}=\delta_{2}$, they interfere destructively two by two.

4.3 INTERPRETATION IN TERMS OF RAYLEIGH SCATTERING, STIMULATED AND SPONTANEOUS RAMAN SCATTERING. - When $\Omega_{2}=0$, the only scattering path which remains open is the one passing through $\left|\varphi_{e}\right\rangle=\left|e,(N)_{2}\right\rangle$ (Path b of Fig. 4). This is due to the fact that, during the absorption of the incident $\omega_{L 1}$ photon, the number $N$ of $\omega_{L 2}$ photons does not change, so that the total system cannot go, by an interaction with the $\omega_{L 1}$ photon, from $\left|g_{1},(N)_{2}\right\rangle$ to $\left|\varphi_{2}\right\rangle=\left|g_{2},(N+1)_{2}\right\rangle$. Furthermore, the dipole moment operator appearing in the interaction hamiltonian $V$ has no matrix element between $g_{1}$ and $g_{2}$. It is only because the dressed state

$\overline{\left|\varphi_{2}\right\rangle}$ contains a small admixture of $\left|\varphi_{e}\right\rangle=\left|e,(N)_{2}\right\rangle$ (see Eq. (4.11b)), that the system can go from $\left|g_{1},(N)_{2}\right\rangle$ to $\overline{\left|\varphi_{2}\right\rangle}$ when $\Omega_{2} \neq 0$. Such a contamination can be described in terms of virtual absorptions and emissions of $\omega_{L 2}$ photons by the atom in $g_{2}$. So, we expect that the scattering path passing through $\overline{\left|\varphi_{2}\right\rangle}$ could be described in the basis of bare states provided that we introduce, in addition to the $\omega_{L 1}$ and $\omega$ photons which are the only ones to appear in figures 3 and 4 , extra $\omega_{L 2}$ photons to describe such contamination effects.

To carry out such a program, we come back to equation (4.13), and we calculate each term of the bracket, at the lowest order in $\Omega_{2}$ where it is not vanishing. The first term, which corresponds to the path remaining open when $\Omega_{2}=0$, can be calculated to order zero in $\Omega_{2}$. We then have $\alpha \simeq\left\langle\varphi_{e} \overline{\mid \varphi_{e}}\right\rangle \simeq 1, z_{1} \sim-i \Gamma / 2$, so that :

$$
\alpha\left\langle\varphi_{e} \overline{\mid \varphi_{e}}\right\rangle \frac{1}{\delta_{1}-z_{\mathrm{I}}} \simeq \frac{1}{\delta_{1}+i \frac{\Gamma}{2}}
$$

The second term requires more caution. Both $\beta$ and $\left\langle\varphi_{e} \overline{\left|\varphi_{2}\right\rangle}\right.$ vanish when $\Omega_{2}=0$. They appear only to first order in $\Omega_{2}$ and, at this order, one gets from (4.11) :

$$
\beta \simeq\left\langle\varphi_{e} \overline{\mid \varphi_{2}}\right\rangle \simeq \frac{\Omega_{2} / 2}{\delta_{2}+i \frac{\Gamma}{2}}
$$




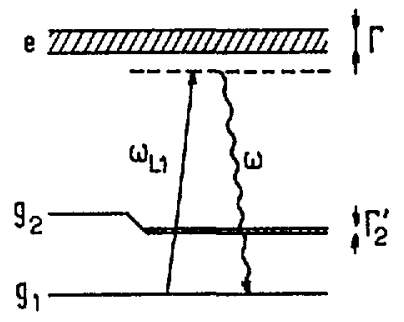

a)

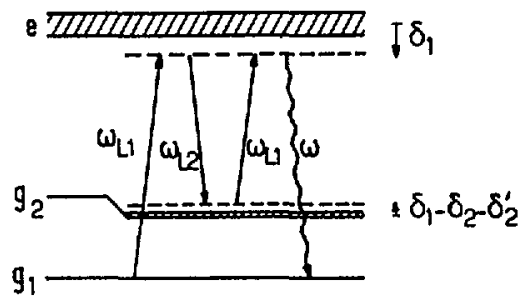

b)

Fig. 5. - Diagrammatic representation of the scattering process in terms of bare states including the light shifts and radiative broadenings due to the $\omega_{L 2}$ photons. a) Rayleigh scattering process from $g_{1}$ (absorption of $\omega_{L 1}$, followed by the spontaneous emission of $\omega$ ). b) Stimulated Raman process bringing the atom from $g_{1}$ to $g_{2}$ (absorption of $\omega_{L 1}$ and stimulated emission of $\omega_{L 2}$ ) followed by a spontaneous Raman process bringing the atom from $g_{2}$ to $g_{1}$ (absorption of $\omega_{L 2}$ and spontaneous emission of $\omega$ ).

so that, using (4.2), we can write :

$$
\begin{aligned}
\beta\left\langle\varphi_{e} \overline{\left|\varphi_{2}\right\rangle} \frac{1}{\delta_{1}-z_{\mathrm{II}}}\right. & \simeq \frac{\left(\Omega_{2} / 2\right)^{2}}{\left(\delta_{2}+i \frac{\Gamma}{2}\right)^{2}} \frac{1}{\delta_{1}-\delta_{2}-\delta_{2}^{\prime}+i \frac{\Gamma_{2}^{\prime}}{2}} \\
& \simeq \frac{\left(\Omega_{2} / 2\right)^{2}}{\left(\delta_{1}+i \frac{\Gamma}{2}\right)^{2}} \frac{1}{\delta_{1}-\delta_{2}-\delta_{2}^{\prime}+i \frac{\Gamma_{2}^{\prime}}{2}}
\end{aligned}
$$

In going from the first line of (4.16) to the second line, we have used the fact that, due to the second denominator the whole expression is large only when $\delta_{1} \simeq \delta_{2}-\delta_{2}^{\prime}$, so that we can replace in the first denominator, which gives rise to slow variations (becausè of the large $i \Gamma / 2$ term), $\delta_{2}$ by $\delta_{1}+\delta_{2}^{\prime} \simeq \delta_{1}$. Inserting (4.14) and (4.16) into (4.13), we finally get :

$$
T_{\mathrm{f}} \simeq T_{\mathrm{f}}^{\mathrm{I}}+T_{\mathrm{f}}^{\mathrm{II}}
$$

with

$$
\begin{aligned}
& T_{\mathrm{fi}}^{\mathrm{I}} \simeq \frac{\hbar \Omega}{2} \frac{1}{\hbar\left(\delta_{1}+i \frac{\Gamma}{2}\right)} \frac{\hbar \Omega_{1}}{2} \\
& T_{\mathrm{fl}}^{\mathrm{II}} \simeq \frac{\hbar \Omega}{2} \frac{1}{\hbar\left(\delta_{1}+i \frac{\Gamma}{2}\right)} \frac{\hbar \Omega_{2}}{2} \frac{1}{\hbar\left(\delta_{1}-\delta_{2}-\delta_{2}^{\prime}+i \frac{\Gamma_{2}^{\prime}}{2}\right)} \frac{\hbar \Omega_{2}}{2} \frac{1}{\hbar\left(\delta_{1}+i \frac{\Gamma}{2}\right)} \frac{\hbar \Omega_{1}}{2}
\end{aligned}
$$

We can now give a diagrammatic representation of the amplitudes (4.18a) and (4.18b) in terms of bare states $e, g_{1}, g_{2}$, keeping however the radiative widths and shifts of these levels (for $e$, we neglect $\delta_{2}^{\prime}$ and $\Gamma_{2}^{\prime}$ in comparison with $\Gamma$, to be consistent with the approximation made in (4.16)). Reading (4.18a) and (4.18b) from right to left, we associate a photon absorption or emission with each Rabi frequency term $\hbar \Omega_{1} / 2, \hbar \Omega_{2} / 2$ or $\hbar \Omega / 2$, and an intermediate state with each energy denominator, such an energy denominator giving the energy defect between the energy of the initial state and the energy of the intermediate state.

We first consider (4.18a) (Fig. 5a). Starting from $g_{1}$ the atom absorbs the $\omega_{L 1}$ photon $\left(\hbar \Omega_{1} / 2\right.$ term) and jumps into $e$, the energy defect being $\hbar\left(\delta_{1}+i \frac{\Gamma}{2}\right)$, and then falls back into $g_{1}$ by emission of the fluorescence photon $\omega(\hbar \Omega / 2$ term). Such a process is nothing but ordinary (near resonant) Rayleigh scattering. 
The amplitude ( $4.18 \mathrm{~b}$ ) corresponds to the four-photon process represented in figure $5 \mathrm{~b}$. Here also, the atom starts from $g_{1}$ and absorbs the $\omega_{L 1}$ photon $\left(\hbar \Omega_{1} / 2\right.$ term) to go into $e$ with an energy defect $\hbar\left(\delta_{1}+i \frac{\Gamma}{2}\right)$. But now, from $e$, the atom jumps into $g_{2}$ by stimulated emission of one $\omega_{L 2}$ photon $\left(\hbar \Omega_{2} / 2\right.$ term), the new energy defect becoming $\hbar\left(\delta_{1}-\delta_{2}-\delta_{2}^{\prime}+i \frac{\Gamma^{\prime}}{2}\right)$. Then, the atom absorbs one photon $\omega_{L 2}\left(\hbar \Omega_{2} / 2\right.$ term) to return to $e$, with the same energy defect $\hbar\left(\delta_{1}+i \frac{\Gamma}{2}\right)$ as before, and finally falls back into $g_{1}$ by spontaneously emitting the fluorescence photon $\omega\left(\hbar \Omega / 2\right.$ term). The amplitude $T_{\mathrm{f}}^{\mathrm{II}}$ thus describes a sequence of two Raman processes, one stimulated Raman process which brings the atom from $g_{1}$ to $g_{2}$, followed by a spontaneous Raman processes, which brings back the atom from $g_{2}$ to $g_{1}[15]$. The amplitude of such a combined process becomes very large when the second intermediate state is resonant, i.e. when $\delta_{1}=\delta_{2}+\delta_{2}^{\prime}$. The width of the resonance is the width $\Gamma_{2}^{\prime}$ of $g_{2}$.

The expression (4.18b) of $T_{\mathrm{fi}}^{\mathrm{II}}$ looks like a perturbative expression taken from a Born expansion of the scattering amplitude. Actually this is not the case. The presence of $\delta_{2}^{\prime}$ and $\Gamma_{2}^{\prime}$ in the second energy denominator results in the fact that $(4.18 \mathrm{~b})$ is a non-perturbative scattering amplitude. The presence of $\Omega_{2}^{2}$ in the numerator of (4.18b) does not imply that $T_{\mathrm{f}}^{\mathrm{II}}$ is of order 2 in $\Omega_{2}$. When $\delta_{1}=\delta_{2}+\delta_{2}^{\prime}$, the second energy denominator of $(4.18 \mathrm{~b})$ becomes equal to $i \Gamma_{2}^{\prime} / 2$, which is also proportional to $\Omega_{2}^{2}$, so that $T_{\mathrm{fi}}^{\mathrm{Il}}$ is then of order 0 in $\Omega_{2}$, and can become on the order of, or even larger than $T_{\mathrm{f}}^{\mathrm{I}}$. It would be also wrong to add to (4.17) amplitudes involving more $\omega_{L 2}$ photons. Equation (4.17) results from the exact expression (4.13), which already contains a resummation of the perturbation series.

\section{Conclusion : Connection with Fano profiles.}

In the low saturation limit considered in the previous section, $\Gamma \gg \Gamma_{2}^{\prime}$, and the excited state $e$ appears as a continuum, in comparison with the narrow levels $g_{1}$ and $g_{2}$. Figures $5 \mathrm{a}$ and $5 \mathrm{~b}$ show that there are two distinct paths for going from the discrete state $g_{1}$ to the "continuum" e.

The first path appears in figure 5a. It corresponds simply to the absorption of the photon $\omega_{L 1}$ which brings directly the atom from $g_{1}$ to $e$. The second path, appearing in Fig. $5 \mathrm{~b}$, is a three-photon process going through $g_{2}$. A stimulated Raman process (absorption of $\omega_{L_{1}}+$ stimulated emission of $\omega_{L_{2}}$ ) brings the atom from $g_{1}$ to $g_{2}$, and then the absorption of one $\omega_{L 2}$ photon brings the atom from $g_{2}$ into the "continuum" $e$. This second path, which passes through a narrow discrete state $g_{2}$, interferes with the first one, which is a direct path towards the continuum. Such a situation leads to Fano profiles.

To check this point more carefully, we come back to equation (3.20), and, using (4.2) and (4.3), we rewrite the first line of this equation as :

$$
T_{\mathrm{fi}}=\frac{\hbar \Omega_{1} \Omega}{4\left[\delta_{1}+\delta_{2}^{\prime}+i \frac{\Gamma-\Gamma_{2}^{\prime}}{2}\right]} \frac{\delta_{1}-\delta_{2}}{\delta_{1}-\delta_{2}-\delta_{2}^{\prime}+i \frac{\Gamma_{2}^{\prime}}{2}}
$$

Because of the presence of $i \Gamma / 2$ in the denominator, the first fraction of (5.1) varies very slowly with $\delta_{1}$, in comparison with the second fraction. We now transform the second fraction of (5.1), which is the one giving rise to the rapid variations of $T_{\mathrm{f}}$ around $\delta_{1}=\delta_{2}+\delta_{2}^{\prime}$ by introducing the reduced variables:

$$
\varepsilon=\frac{\delta_{1}-\delta_{2}-\delta_{2}^{\prime}}{\Gamma_{2}^{\prime} / 2}
$$




$$
q=\frac{\delta_{2}^{\prime}}{\Gamma_{2}^{\prime} / 2}=\frac{2 \delta_{2}^{\prime}}{\Gamma_{2}^{\prime}}=\frac{2 \delta}{\Gamma}
$$

This allows one to write $\left|T_{\mathrm{fi}}\right|^{2}$ as :

$$
\left|T_{\mathrm{f}}\right|^{2}=\frac{\hbar^{2} \Omega_{1}^{2} \Omega^{2}}{16\left[\left(\delta_{1}+\delta_{2}^{\prime}\right)^{2}+\frac{\left(\Gamma-\Gamma_{2}^{\prime}\right)^{2}}{4}\right]} \frac{(\varepsilon+q)^{2}}{\varepsilon^{2}+1}
$$

The second fraction of (5.4) is typical of a Fano profile with parameter $q$.

We have thus proven that the narrow structure of figure 2 is a Fano profile, and we have identified in figure 5 the two interfering pathways which give rise to such a profile.

\section{Acknowledgements.}

We thank G. Grynberg for stimulating discussions.

Cet article est un témoignage de notre admiration pour l'oeuvre scientifique de Pierre Jacquinot et de notre reconnaissance pour le travail qu'il a accompli pour le développement de la Recherche Scientifique en France et dont nous avons tous bénéficié.

\section{References}

[1] Alzetta G., Gozzini A., MoI L. and OrRiols G., Nuovo Cim. B36 (1976) 5.

[2] GRAY H.R., Whitley R.M. and STROUd C.R. Jr, Opt. Lett. 3 (1978) 218.

[3] ARIMondo E. and OrRIols G., Nuovo Cim. Lett. 17 (1976) 133.

[4] Radmore P.M. and KNight P.L., J. Phys. B14 (1981) 573.

[5] JaNiK G., Nagourney W., Dehmelt H., J.O.S.A. B2 (1985) 1251.

[6] Dalibard J., Reynaud S., Cohen-tannoudji C., in Interaction of Radiation with Matter, a Volume in honour of A. Gozzini, Scuola Normale Superiore, Pisa (1987).

[7] Thomas J.E., Hemmer P.R., Ezekiel S., Leiby C.C., Picard R.H., Willis C.R., Phys. Rev. Lett. 48 (1982) 867.

[8] Aspect A., Arimondo E., Kaiser R., Vansteenkiste N. and Cohen-tannoudji C., Phys. Rev. Lett. 61 (1988) 826 ; J.O.S.A. B6 (1989) 2112.

[9] Gaubatz U., Rudecki P., Schiemann S. and Bergmann K., J. Chem. Phys. 92 (1990) 5363.

[10] Imamoglu A., Field J.E., Harris S.E., Phys. Rev. Lett. 66 (1991) 1154.

[11] Fano U., Phys. Rev. 124 (1961) 1866.

[12] Armstrong L. Jr., Beers B.L. and Feneuille S., Phys. Rev, A12 (1975) 1903.

[13] Knight P.L., Comments At. Mol. Phys. 15 (1984) 193.

[14] Cohen-Tannoudji C., Dupont-roc J. and GRYnBerg G., Processus d'Interaction entre Photons et Atomes (Intereditions et Editions du CNRS, Paris, 1988). English translation : AtomPhoton Interactions. Basic Processes and Applications (Wiley, New-York, 1992).

[15] Other examples of interferences between different pathways, one of them involving a stimulated Raman process may be found in : Grynberg G. and Berman P.R., Phys. Rev. A41 (1990) 2677. In the problem considered in this paper, the dissipative process is not spontaneous emission, but collisions. 